\title{
Maxillary arch expansion along with fixed mechanotherapy using different expansion devices
}

\author{
Medha Lakhanam ${ }^{1}$, Tripti Tikku ${ }^{2}$, Rohit Khanna ${ }^{3}$, Rana Pratap Maurya $^{4}$, Snehlata Verma ${ }^{5}$, \\ Kamna Srivastava, ${ }^{6, *}$
}

${ }^{1}$ Junior Resident, ${ }^{2}$ Professor and HOD, ${ }^{3}$ Professor, ${ }^{4-6}$ Reader, Dept of Orthodontics and Dentoficial Orthopedics, Babu Banarasi Das College of Dental Sciences, Lucknow, Uttar Pradesh, India

*Corresponding Author:

Email: amitn99@gmail.com

\begin{abstract}
Malocclusions can exist in all the three planes of space i.e in antero - posterior, vertical and transverse plane. Transverse discrepancies can manifest as dental, skeletal or functional discrepancies. Maxillary expansion is one of the most common treatment modality for arch length augmentation, posterior crossbite correction and removing cross arch interferences.

Skeletal correction is done by orthopedic expansion or surgical expansion. Rapid Maxillary Expansion and Head Gears, produces orthopedic correction. Severe skeletal discrepancies require surgical correction of underlying skeletal deformity by using SARPE (Surgically Assisted Rapid Palatal Expansion) or surgical expansion of palatal shelves by midline osteotomies.

Dental correction can be done using Rapid expansion in growing children and by slow expansion using Quad Helix, W Arches, Coffin Spring and certain modification of Jack screw appliances in other cases.

In patients treated with fixed mechanotherapy expansions can not only be achieved by Arch wires, auxillary Jockey arches, cross elastics etc. but also by slow or rapid expansion devices that can be amalgamated with fixed orthodontic treatment.

In this article, the use of slow maxillary expansion appliances in treatment of the three patients with transverse maxillary deficiency and undergoing fixed mechanotherapy will be discussed.
\end{abstract}

Keywords: Expansion.

\section{Introduction}

Malocclusions can exist in all the three planes of space i.e in antero-posterior, vertical and transverse plane. Transverse discrepancies can manifest as dental, skeletal or functional discrepancies. Maxillary arch expansion is one of the common non extraction treatment modality for arch length augmentation, posterior crossbite correction and removing cross arch interferences.

Skeletal correction is done by orthopedic expansion or surgical expansion. Rapid Maxillary Expansion and Head Gears, produces orthopedic correction. Severe skeletal discrepancies require surgical correction of underlying skeletal deformity by using SARPE (Surgically Assisted Rapid Palatal Expansion) or surgical expansion of palatal shelves by midline osteotomies.

Dental correction can be done using Rapid expansion in growing children and by slow expansion using Quad Helix, W Arches, Coffin Spring and certain modification of Jack screw appliances in other cases.

Slow expansion devices can be amalgated with fixed mechanotherapy to achieve dentoalveolar expanxion which reduces the total duration of treatment time. Overhanging of palatal cusp of posterior teeth that may occurs due to dentoalveolar expansion with slow exapansion devices can be simaultaneously corrected by incorporating buccal root torque in the main arch wire.
In this presentation, the use of slow maxillary expansion appliances with fixed mechanotherapy will be shown in two patients having transverse maxillary deficiency.

\section{Case-I}

A 27-year-old male patient was referred to department of orhodontics with chief complaint of spacing in maxillary and mandibular anterior region. On examination, the patient had a convex profile, increased interlabial gap, tongue thrust, unilateral posterior crossbite on right side and asymmetric maxillary arch. All the pre treatment records (Lateral cepalogram, O.P.G, study models and extraoral and intraoral photographs) were taken and analysed (Fig. 1A).

Based on the clinical diagnosis non extraction orthodontic treatment was planned aiming to correct posterior crossbite on right side and closure of spaces present in maxillary and mandibular anterior region.

To correct the posterior unilateral crossbite removable appliance with jack screw was designed and closure of spaces was planned. Appliance base plate was cut in the posterior region in such a manner to produce unilateral expansion of posterior segment of right side (Fig. 1B) whereas remaining portion of the base plate served as anchorage unit, from palatal aspect. Buccal root torque was incorporated in main arch wire when reached to $17 \mathrm{X} 25 \mathrm{SS}$ wire after sequential change of alignment wires. 


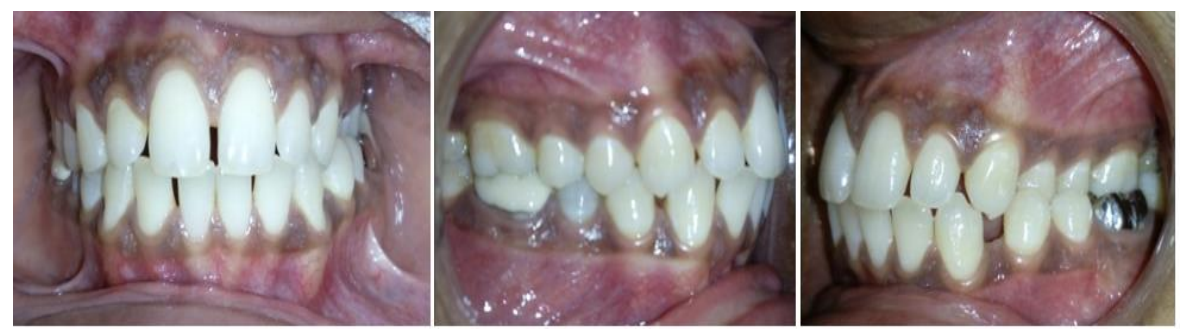

Fig. 1(A): Pretreatment intraoral photographs showing unilateral crossbite on left side.

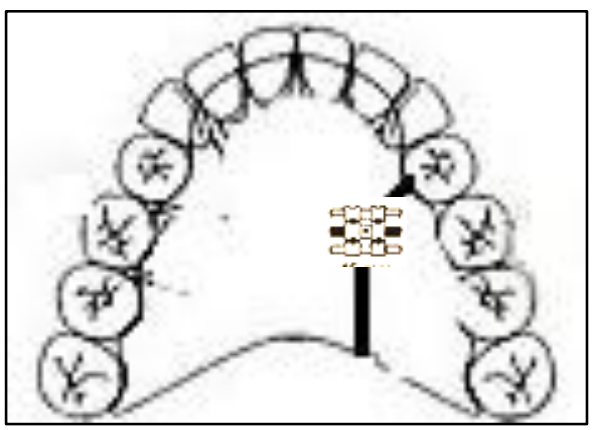

Fig. 1(B): The slow expansion jack screw appliance

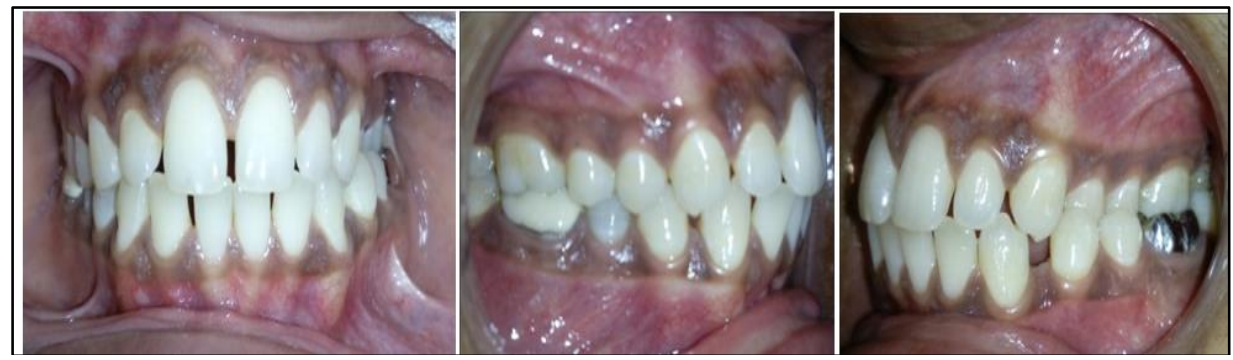

Fig. 1 (B): Pretreatment intraoral photographs showing unilateral crossbite on right side

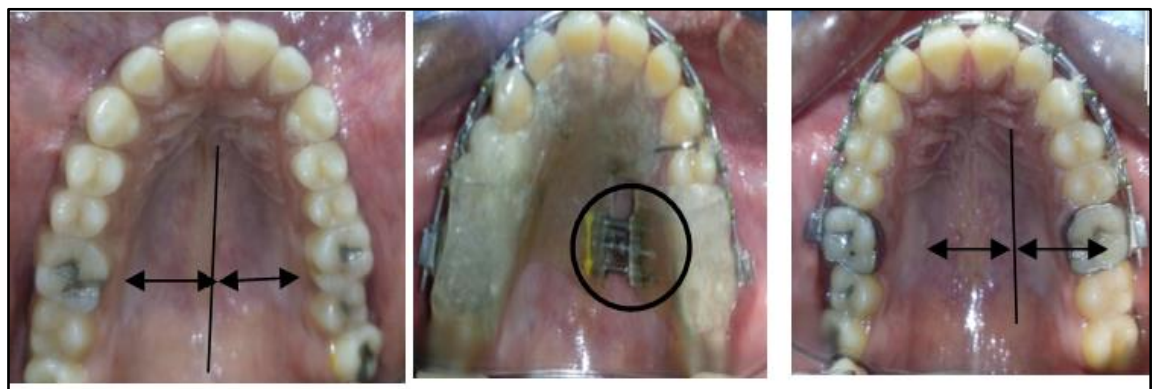

Fig. 1(C): Showing Maxillary arch expansion on right side

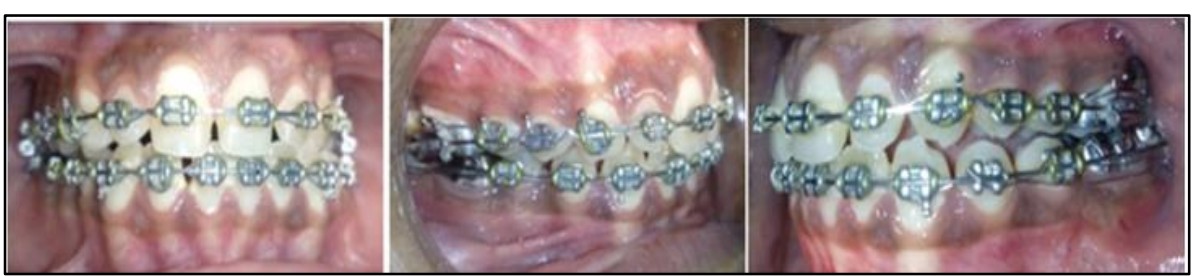

Fig. 1(D): The mid-stage treatment intraoral photographs depicting correction of posterior crossbite on right side

\section{Treatment Progress}

Activation of expansion screw was performed 1quarter turns $(0.25 \mathrm{~mm})$ onceper week until the desired overexpansion was achieved, evaluated by the crossbite correction (after 4 months) simultaneously alignment of the arch wire with $0.016,0.018 \mathrm{Ni}-\mathrm{Ti}$ wires and the 
correction was stablised by buccal crown torque was incorporated in $17 \times 25 \mathrm{SS}$ wire. Simulataneous alignment and leveling and closure of spaces in maxillary and mandibular arches was done using sequential ire $(0.016,0.018 \mathrm{Ni}-\mathrm{Ti}$ and $0.016 \mathrm{X} 0.025 \mathrm{SS}$ wire). $4 \mathrm{~mm}$ expansion in 4 months along with overcorrection of crossbite for preventing relapse chances.

\section{Case II}
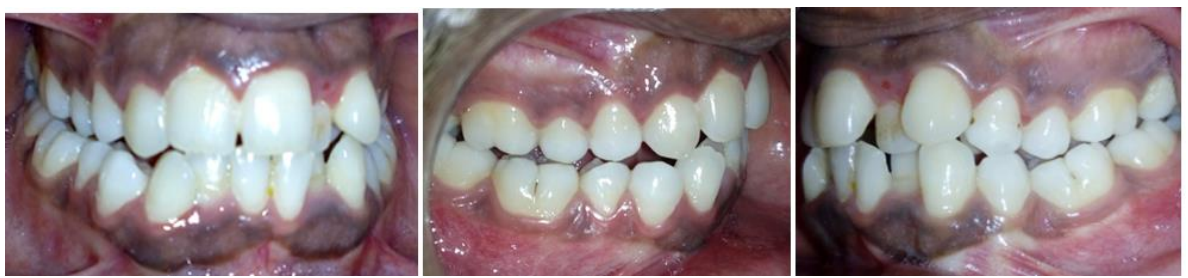

Fig. 2(A): Pretreatment intraoral photographs showing anterior crossbite on left side and bilateral posterior crossbite

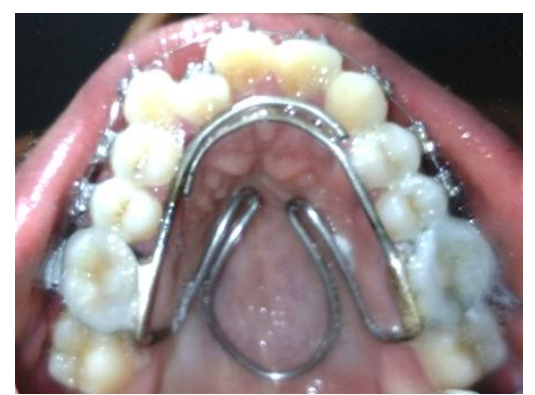

Fig. 2(B): The modified coffin spring appliance for correction of anterior crossbite
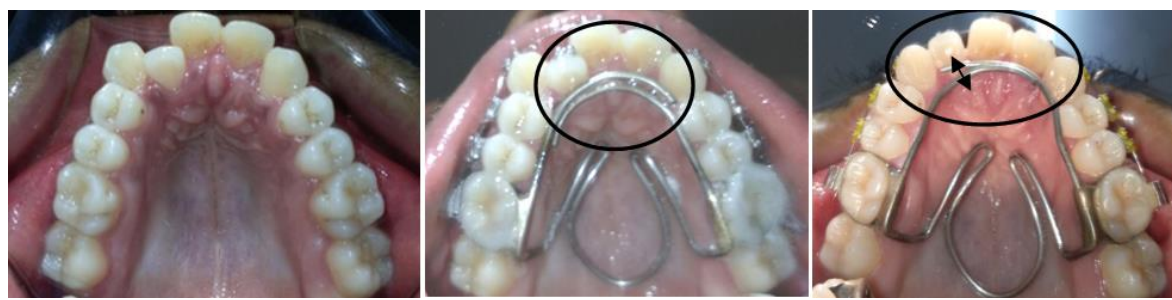

Fig 2(C): Maxillary arch photographs: before, during and after expansion showing maxillary anterior expansion and correction of right lateral incisor
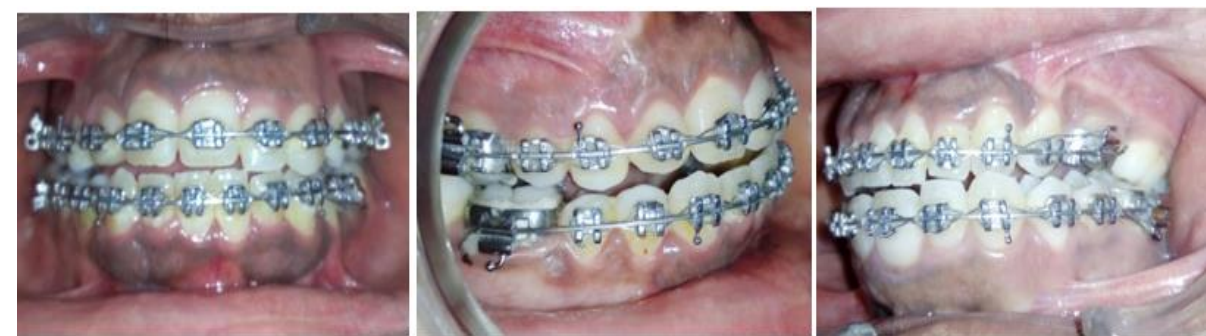

Fig. 2(D): The mid-stage treatment intraoral photographs

\section{Treatment Progress}

Alignment and leveling of maxillary arch was started with $0.014 \mathrm{NiOTi}$ arch wire. On the palatal aspect modifies coffin spring was soldered on maxillary first molar bands and cemented. Activation of coffin spring was performed on scheduled monthly appointment and the desired expansion was achieved, evaluated by the crossbite correction of posterior segment and adequate space achieved for lateral incisor alongwith fixed mechanotherapy. 


\section{Conclusion}

Expansion in maxilla and maxillary dentition may be accomplished in numerous ways. The type of skeletal and dental problem greatly influences the type of expansion chosen and the type of expansion selected can greatly facilitate the overall treatment objectives.

In both these cases slow expansion appliances were employed alongwith fixed mechanotherapy. The treatment goal was achieved by the activated appliance in coordination with alignment and leveling stages of fixed mechanotherapy. These slow expansion appliances help in expansion of the arches, while fixed mechanotherapy facilitates the bodily movement of tooth by incorporating buccal root torques in the ss arch wire. This combination reduces the duration of treatment.

\section{References}

1. Agrwal A, Mathur R. Maxillary Expansion. Int J Clin Pediatr Dent. 2010;3(3):139-46.

2. Handelman C.S. Adult Nonsurgical Maxillary and Concurrent Mandibular Expansion; Treatment of Maxillary Transverse Deficiency and Bidental Arch Constriction. Semin Orthod. 2012;18:134-51.

3. Mutinelli S, Cozzani M. Rapid maxillary expansion in contemporary orthodontic literature. APOS Trends Orthod. 2016;6:129-36.

4. Koudstaal, M. J., Poort, L. J., van der Wal, K. G. H., Wolvius, E. B., Prahl-Andersen, B., \& Schulten, A. J. M. Surgically assisted rapid maxillary expansion (SARME): a review of the literature. Int J Oral and Maxillofacial Surgy. 34(7), 709-14.

5. Pereira JS, Jacob HB, Locks A, Brunetto M, Ribeiro GLU. Evaluation of the rapid and slow maxillary expansion using cone-beam computed tomography: a randomized clinical trial. Dent Press J Orthod. 2017;22(2):61-68.

6. Hicks, E. P. Slow maxillary expansion. Am. J Orthodontics. 73(2):121-41.

7. Marzban R, Nanda R. Slow Maxillary Expansion with Nickel Titanium, J Clin Orthod. 37(8):431-41.

8. Wonga C.A, Sinclairb P.M, Keimc R.G, Kennedya D.B. Arch dimension changes from successful slow maxillary expansion of unilateral posterior crossbite. Angle Orthod. 2011;81:616-23. 